\title{
Forensic Analysis of Heroin and Cocaine Seizures
}

\author{
Thomas A. Briellmann ${ }^{\star a}$, Franz E. Dussya and Micha G. Bovens ${ }^{\mathrm{b}}$
}

\begin{abstract}
In the Section 'Forensic Chemistry and Toxicology' of the Swiss Society of Legal Medicine the group 'Forensic Chemistry' deals with the analysis of drugs of abuse. Some of the toxicology laboratories of the Swiss Institutes of Forensic Medicine also work in this field. The group organizes its own proficiency tests for heroin, cocaine, THC, and amphetamine/designer drugs. Statistics about the percentages of heroin and cocaine in all drugs of abuse seizures analyzed in the Swiss laboratories are published on a yearly basis as an aid to the police or the public prosecutor service. Members of the Group have prepared, by order and together with the Swiss Accreditation Service (metas), Guidelines for the accreditation of the laboratories performing drug of abuse testing. Laboratories which are equipped with an ion mobility spectrometer perform the analysis of trace amounts of heroin and cocaine on hands, hair, nails, clothes and other materials (e.g. bank notes).
\end{abstract}

Keywords: Cocaine · Forensic analysis · Heroin · Illicit samples · Ion mobility spectrometry

\section{Introduction}

For the past few years the group 'Forensic Chemistry' of the Swiss Society of Legal Medicine (SGRM/SSML) has been working on the subject of the analysis of a variety of drugs of abuse. One of the important topics is thereby the analysis of heroin and cocaine which are two of the most frequently abused drugs in our country. The 'Forensic Chemistry' group is composed of almost all laboratories performing forensic drug analyses in Switzerland. Therefore a good platform for the discussion of all analytical problems is established. Due to the high population, the international airport and the economic importance of Zürich, the Chemistry Department of the Scientific

\footnotetext{
${ }^{*}$ Correspondence: Dr. T.A. Briellmanna

Tel.: +41612673895

Fax: +41612673907

E-Mail: thomas.briellmann@bs.ch

alnstitute of Forensic Medicine

Pestalozzistr. 22

$\mathrm{CH}-4004$ Basel

${ }^{b}$ Chemistry Department, Scientific Forensic Service

Zürich City Police

Zeughausstrasse 11

Postfach

$\mathrm{CH}-8021$ Zürich
}

Forensic Service in Zürich is involved in the highest number of cases followed by the Forensic Laboratories of most of the Institutes of Forensic Medicine and some State Laboratories. The Federal Office for Police Matters and the Federal Office for Public Health join the group meetings as guests.

The aims of the meetings of the 'Forensic Chemistry' group are:

- the harmonization of the analytical procedures in use

- the exchange of professional information

- the elaboration of guidelines

In 2001 the following guidelines were prepared and accepted:

- Guidelines for cannabis (sampling, analysis and interpretation)

- Guidelines for the sampling of illicit drugs of abuse

- Guidelines for quality assurance for the analysis of illicit drugs of abuse

\section{Heroin and Cocaine Seizures}

In 1993 Swiss Federal Law stipulated that not the net-weight of the drug but the content of heroin and cocaine of the seizure is decisive for the court and has therefore to be determined. So, dealing with or possession of more than $12 \mathrm{~g}$ of heroin hydrochloride or more than $18 \mathrm{~g}$ of cocaine leads to more severe punishment. In 2000, according to Swiss government statistics, $372 \mathrm{~kg}$ of heroin and $207 \mathrm{~kg}$ of cocaine were seized in our country [1]. $292 \mathrm{~kg}(79 \%)$ of these heroin seizures and $124 \mathrm{~kg}(60 \%)$ of the cocaine seizures were analyzed in the laboratories. Fig. 1 and 2 show the distribution of some different seizures by weight for the heroin and cocaine samples in the year 2000. These statistics will be prepared on a yearly basis by the 'Forensic Chemistry' group. The differences between the total seizures and the analyzed illicit samples in Switzerland can be explained by the fact that in certain cases the forensic analysis is not ordered by the police or the public prosecutor.

\section{Heroin \\ Appearance}

Most of the illicit heroin samples are brown powders filled in portions of about $5 \mathrm{~g}$ in Minigrip ${ }^{\circledR}$ bags (Fig. 3). Bigger samples consist of brown heroin bricks (Fig. 4). Some special dyes are responsible for the brown coloration of the heroin samples. White heroin is seized only rarely in Switzerland. 

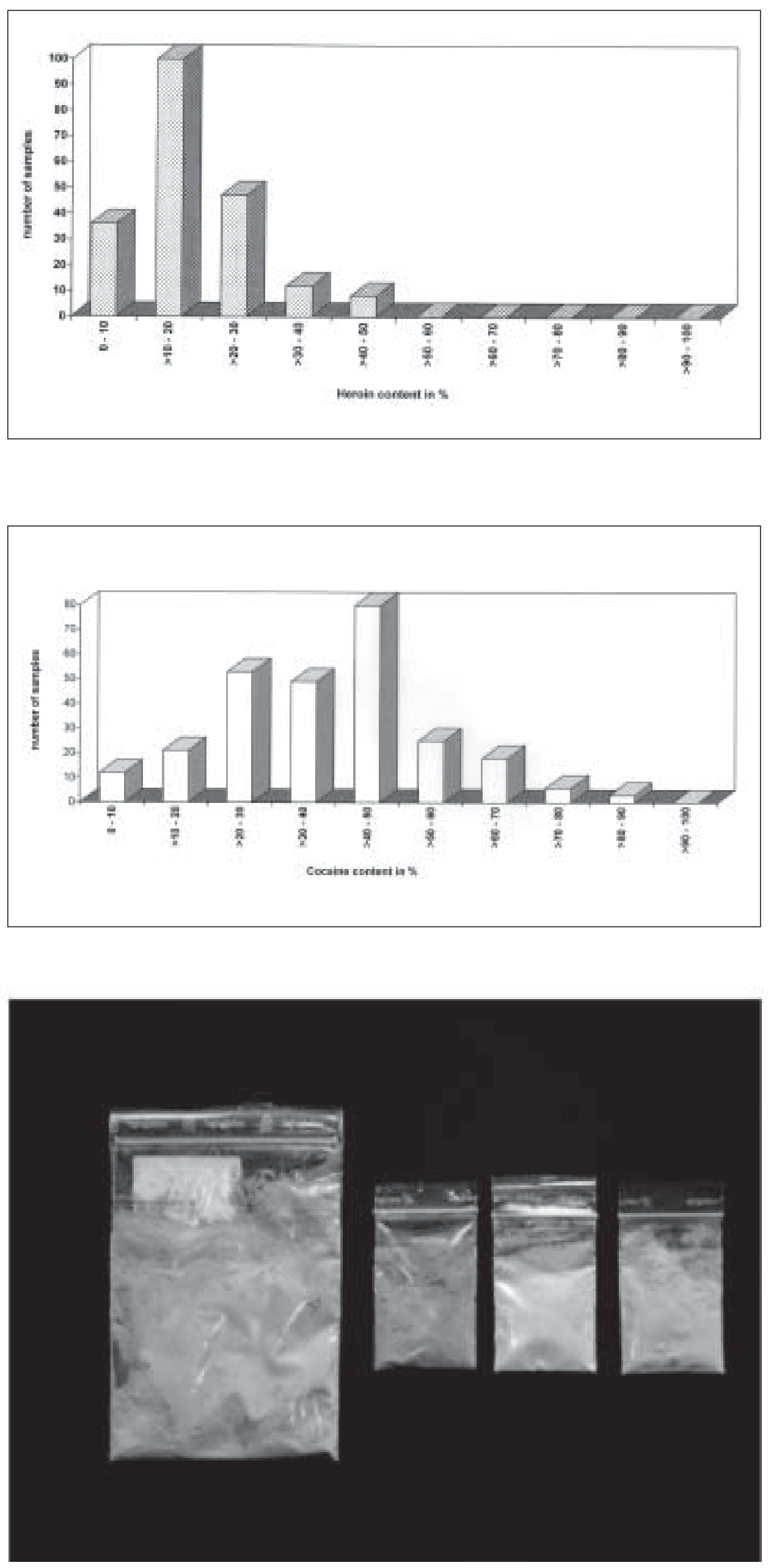

Fig. 1. Heroin content of the heroin seizures between $10 \mathrm{~g}$ and $100 \mathrm{~g}$ in 2000 in Switzerland

Fig. 2. Cocaine content of the cocaine seizures between $1 \mathrm{~g}$ and $10 \mathrm{~g}$ in 2000 in Switzerland

Fig. 3. Heroin samples in Minigrip ${ }^{\circledR}$ bags 


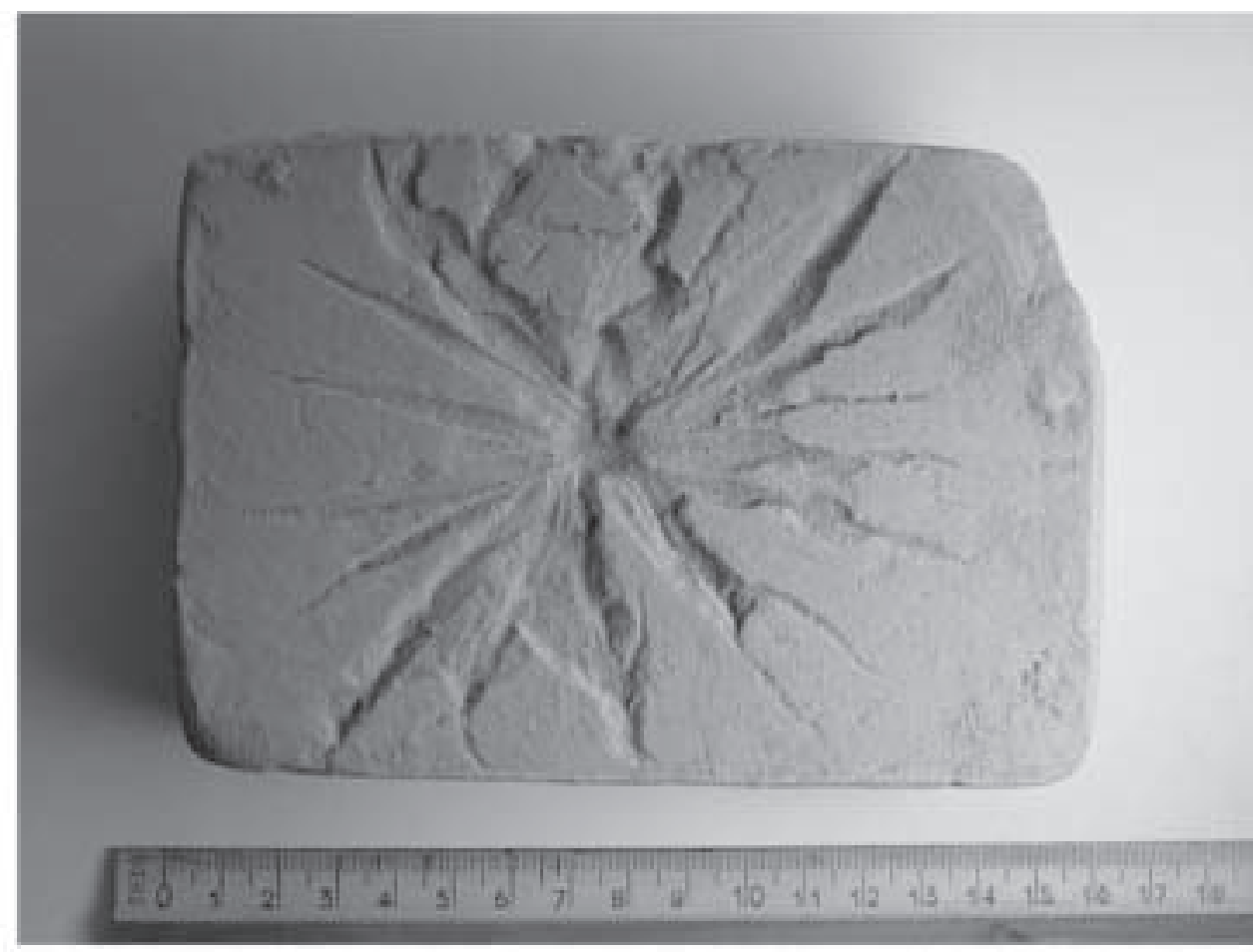

In more than $90 \%$ of cases the illicit heroin samples are seized as base. Heroin hydrochloride samples have become rare.

\section{Sampling}

The sampling of illicit heroin material is undertaken according to the 'Guidelines for the sampling of illicit drugs of abuse' of the 'Forensic Chemistry' group (mentioned above) [2]. It can therefore be guaranteed that a sample taken in one laboratory is as representative as an aliquot sampled in another laboratory.

\section{Methods}

With so-called powder analysis, qualitative and quantitative analyses of heroin can be performed. Qualitative analysis provides not only the proof of the identity of heroin but also the determination of other opium alkaloids and of adulterants (cutting agents). In a typical heroin street sample the following substances can be found [3][4]:

Opium alkaloids: 6-acetylmorphine (monoacetylmorphine) acetylcodeine papaverine noscapine Adulterants: paracetamol caffeine

Gas chromatography-mass spectrometry (GC-MS) is applied as method of choice for the qualitative analysis. But the good old thin-layer chromatography (TLC) or near-infrared spectroscopy (NIR) can serve as fast and cheap screening
Fig. 4. Heroin brick

contents of $<10 \%$ to $35 \%$ can be measured. Fig. 5 shows an example of a chromatogram (GC-FID) of the determination of the heroin content of an illicit heroin sample. One of the crucial points during the heroin analysis is the choice of the ideal solvent for the sample because heroin is thermally unstable in the presence of acidic protons and can react in the injector port of the GC. Therefore aprotic solvents (like chloroform) are the best choice. Another difficulty is the sometimes observed deacetylation of heroin by the adulterant paracetamol in the injector port of the GC, which leads to a slight loss of heroin content and an increase of 6-acetylmorphine. n-Alkanes $\left(\mathrm{C}_{22}\right.$ or $\left.\mathrm{C}_{24}\right)$ are commonly used as internal standards. Some laboratories intro-

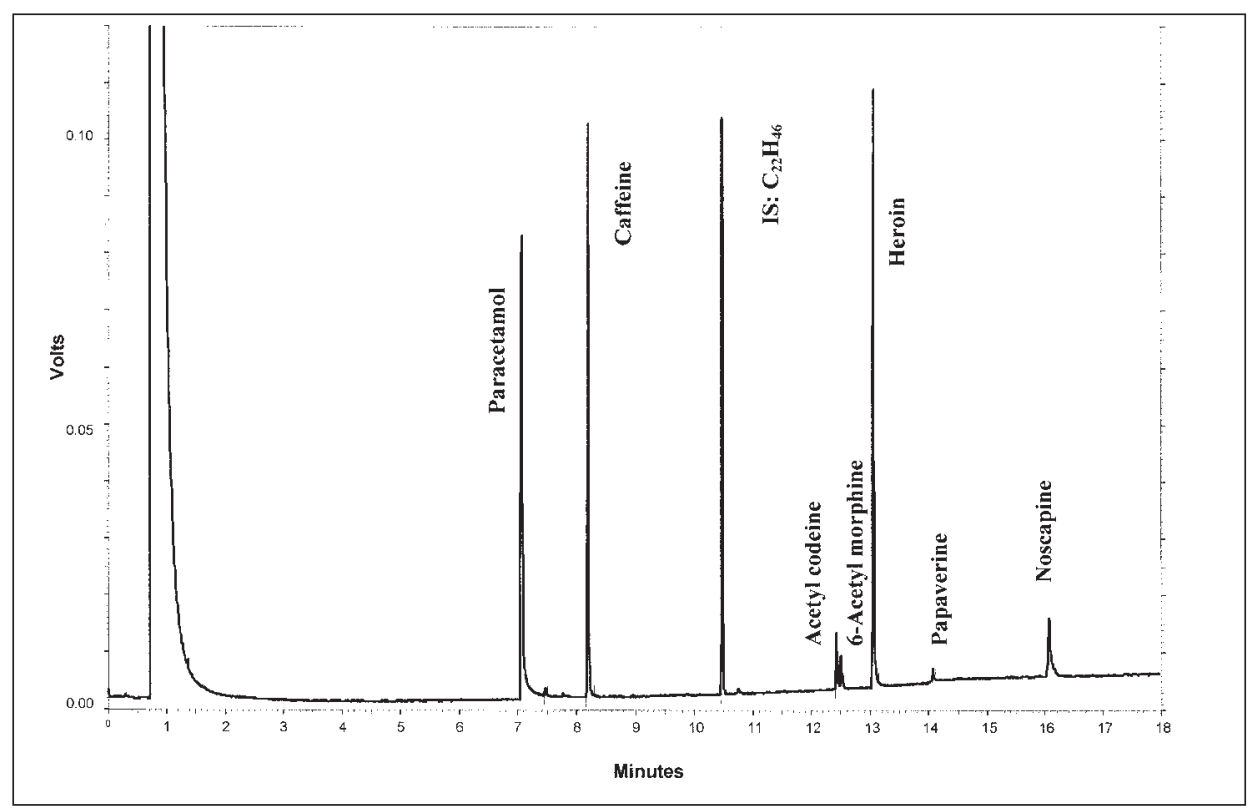

Fig. 5. Chromatogram of the quantification of a heroin street sample 
duce a derivatization step (silylation) before the chromatographic process. HPLC is used as an alternative method in some laboratories.

\section{Comparison of Heroin Samples (Heroin Impurity Profiling)}

The police or prosecutor are increasingly interested in comparing the seized samples, i.e. whether two or more samples have a common origin. Therefore some forensic laboratories use special GC-MS methods to get a 'fingerprint' (impurity profile) of each illicit heroin sample. By comparison of these 'fingerprints' it is possible to distinguish or correlate the samples. Over 20 impurity substances are known, for example meconine, 4-O-acetylthebaol, 6-O,N-diacetylnorcodeine [6].

\section{Cocaine \\ Appearance}

Cocaine samples are usually white or yellowish powders or bricks. The measured cocaine contents are between $20 \%$ and $80 \%$ and are therefore higher than the heroin content of the illicit heroin samples. Usually the cocaine samples are not colored with additional agents as observed in illicit heroin samples. Cocaine (Fig. 6) can be found as the salt (as hydrochloride or more rarely as sulphate) or as base in the form of crack or so-called free-base. In some cases cocaine is smuggled in the stomach or intestine by bodypackers. The cocaine is often pressed into cylindrical bricks and wrapped up in several layers of plastic and fingerstalls (Fig. 7). In this form the cocaine is swallowed or introduced rectally. Sometimes bags, trousers or jackets are soaked with solutions of cocaine and worn in this concealed form beyond the eyes of the customs or the police.

\section{Sampling}

Also for the cocaine cases sampling is performed according to the 'Guidelines for the sampling of illicit drugs of abuse' of the 'Forensic Chemistry' group. Because the cocaine material in containers or compressed bricks is sometimes very hard it has to be pulverized by a mill. If concealed material is analyzed, an extraction process has to be applied first.

\section{Methods}

Although TLC is a good technique for fast screening of cocaine, GC-MS is preferably used in most laboratories for the qualitative analysis. Cocaine too has some special adulterants but not the same

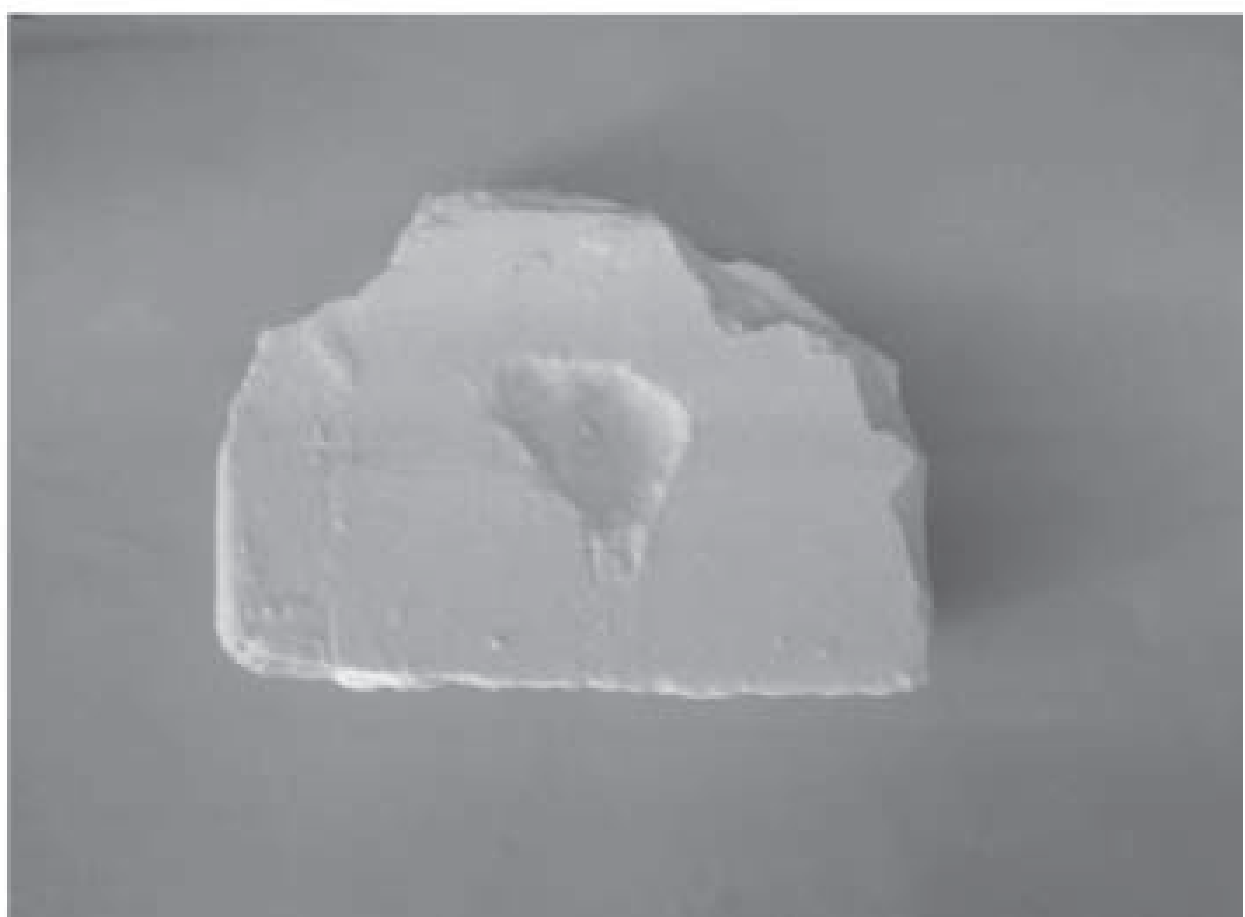

Fig. 6. Cocaine brick

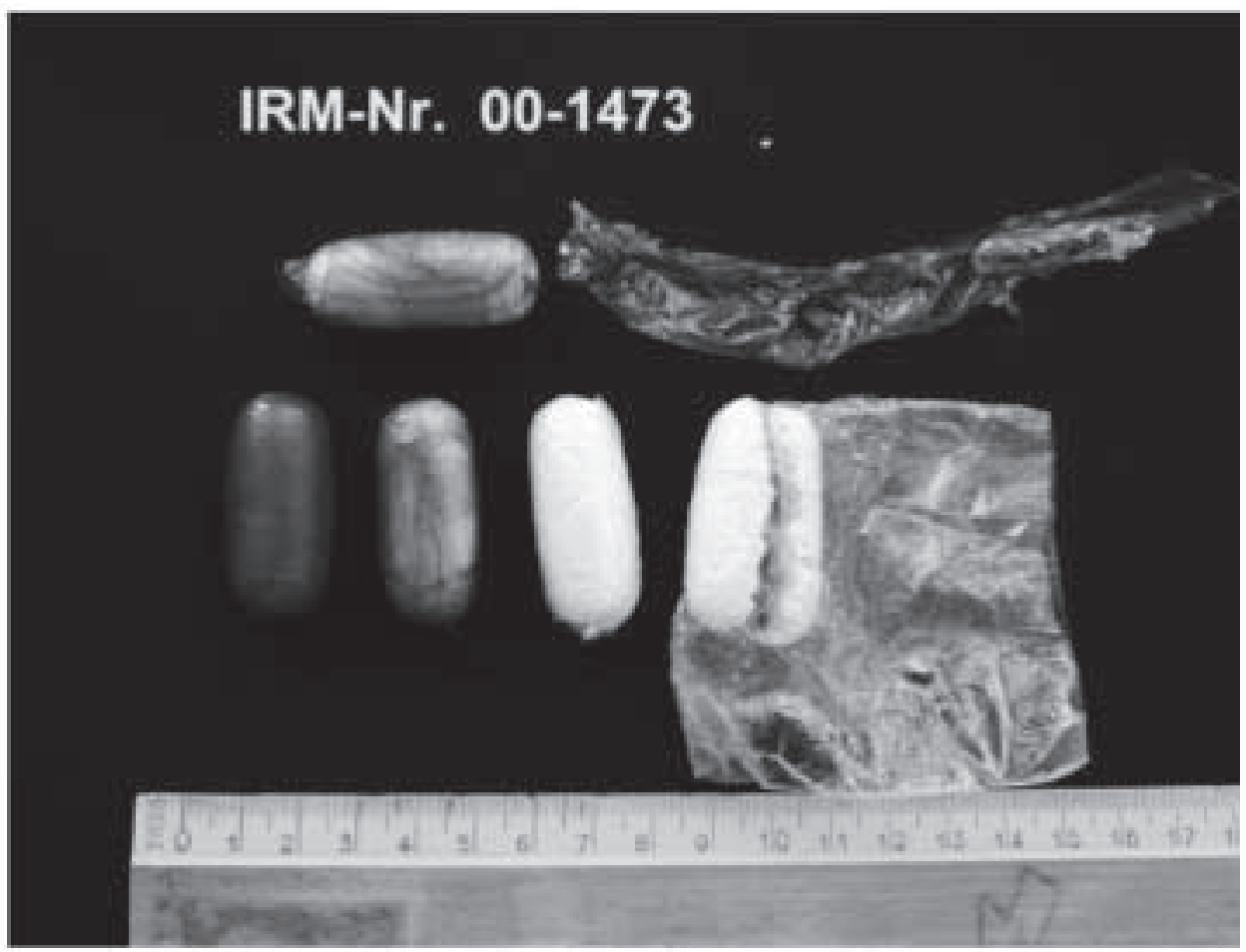

Fig. 7. So-called cocaine containers

as heroin. One can find sugars (e.g. lactose, glucose or saccharose), local anesthetics (e.g. lidocaine or procaine) or inorganic salts (e.g. borate). The coca alkaloid cinnamoylcocaine is found in traces (a few percent) in all illicit cocaine samples.

Quantitative analysis is also performed by GC-FID. Fig. 8 shows an ex- ample for the chromatographic determination of the content of an illicit cocaine sample. There are fewer problems in the chromatography of cocaine compared with the heroin analysis. Also in this method n-alkanes $\left(\mathrm{C}_{22}\right.$ or $\left.\mathrm{C}_{24}\right)$ are used as internal standards. Some laboratories use HPLC instead of GC-FID for the quantification. 


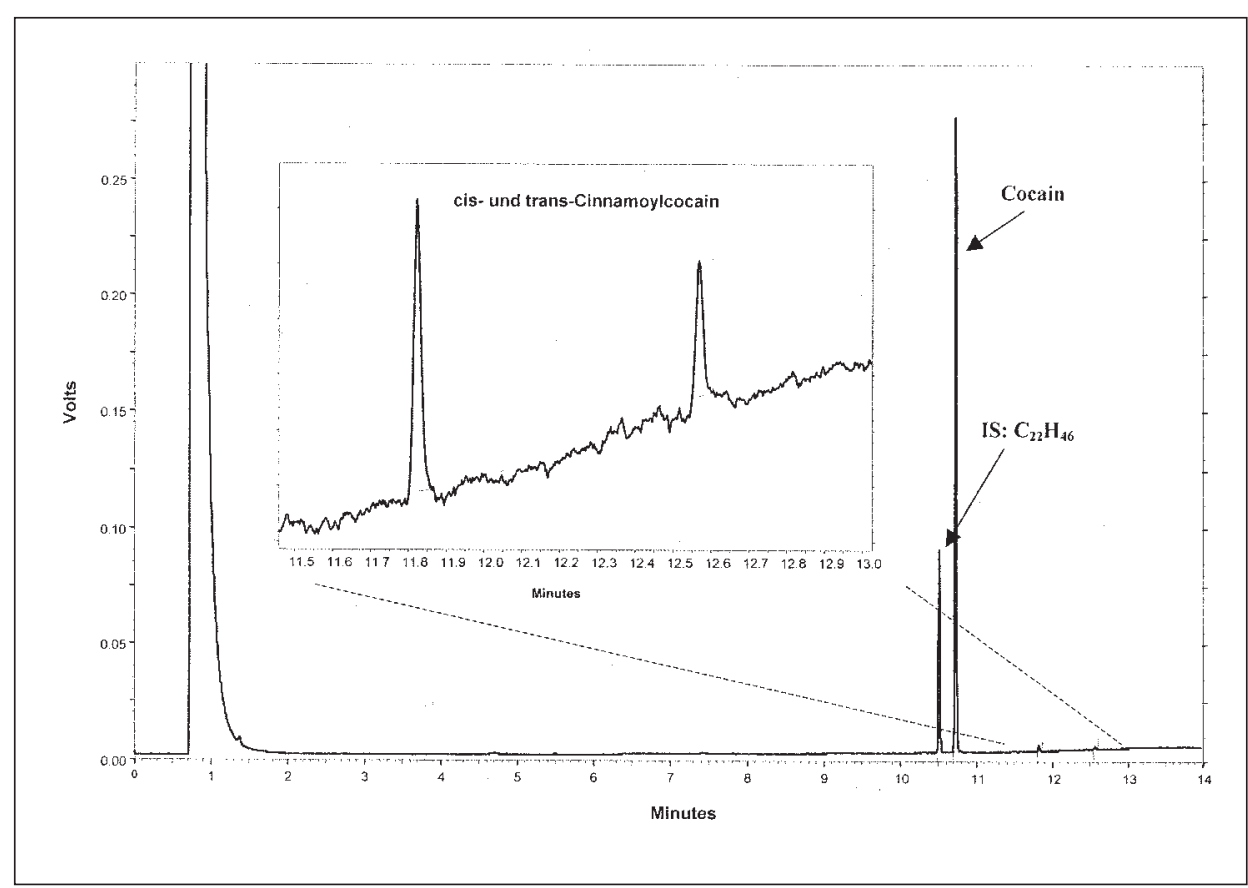

Fig. 8. Chromatogram of the quantification of a cocaine street sample whether the person under suspicion was in contact with illicit drugs. The method is easy to apply and can deliver results in less than one minute. Under the assumption of a correct interpretation of the obtained plasmagrams the method is considered to be cost-effective. A big advantage of the method is the mobility of the equipment itself (Fig. 9). As a consequence there is no need to bring the material or the person under suspicion into the laboratory. A 'field' analysis is possible. If several samples in the same investigation are analyzed, a good picture of the situation is obtained, indicating whether the investigation should be forced or discontinued. IMS spectroscopy is a very sensitive method giving valuable indications of a former presence of more drug material (i.e. on tables, in cars). It is widely accepted by the courts in Switzerland. If necessary the IMS results can be confirmed by GC-MS analysis.

\section{Proficiency Tests}

Some years ago the 'Forensic Chemistry' group started a proficiency test program for the analysis of illegal drugs. Amphetamines, THC, heroin, and cocaine were chosen as analytes and are now determined once a year. The program consists of identification and quantification of illicit drug samples and of samples spiked with drugs of known content. At present participation is still voluntary but should become obligatory in the future. The aim of this quality control is the amelioration and the harmonization of the analytical procedures in the field of forensic drug analysis in the Swiss forensic laboratories. Some of the laboratories participate also in the ENFSI (European Network of Forensic Science Institutes) proficiency test program to get more information about their performance in the international field.

\section{Ion Mobility Spectrometry}

Ion mobility spectrometry (IMS) is a technique which was developed in the seventies and is extensively applied in forensic analysis. In Switzerland it is almost exclusively used to detect traces of heroin or cocaine. The goal of the analyses ordered by the prosecutors or the police is to gain information quickly on

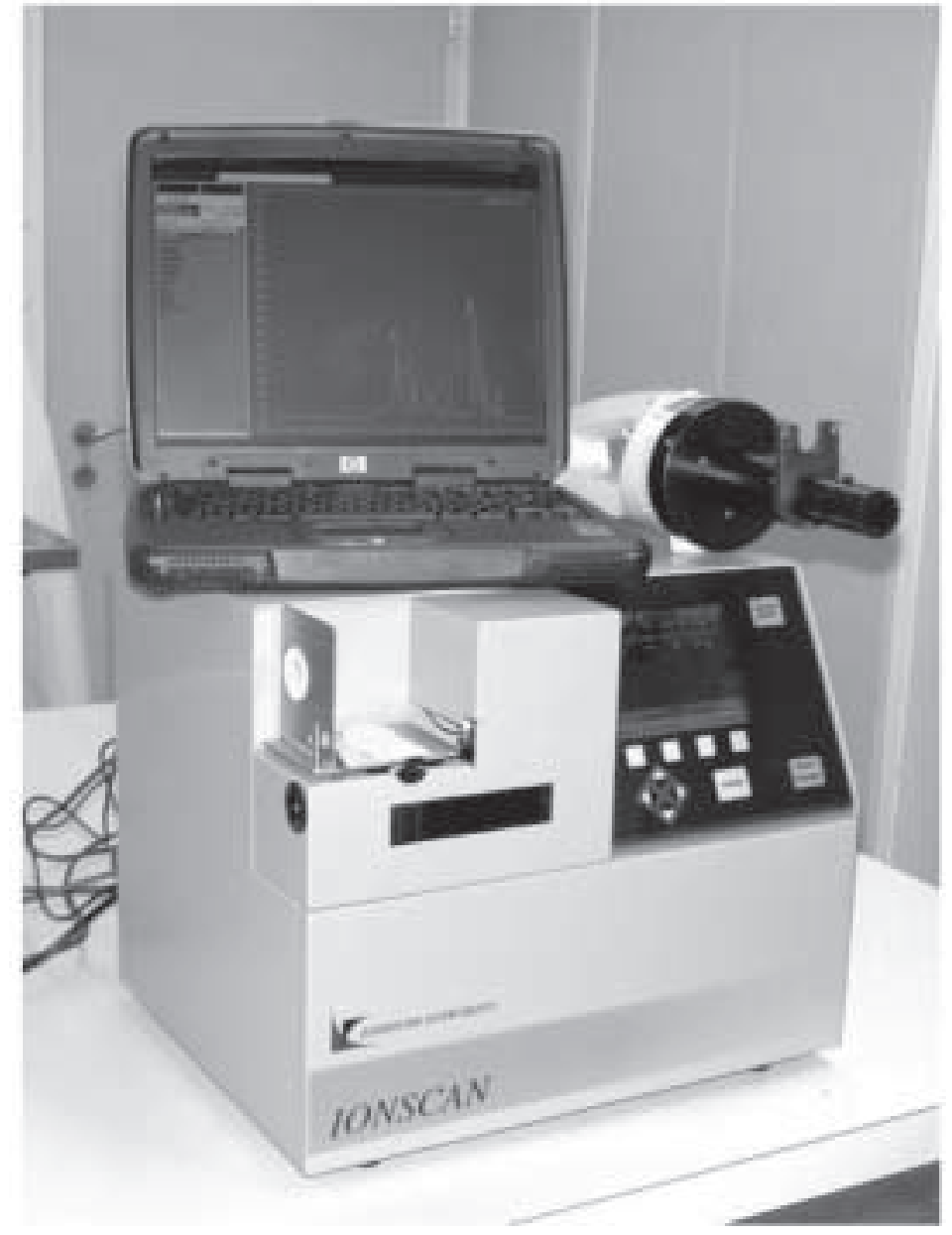

Fig. 9. Ion mobility spectrometry equipment $400 \mathrm{~B}$ 


\section{Procedure}

By a vacuum cleaner the dust is collected on a special Teflon filter and is transferred into the gas phase by application of heat. The molecules are ionized by a ${ }^{63} \mathrm{Ni}$ source emitting $\beta$-radiation. Those ions are accelerated in an electric field against a stream of purified air. The time which an ion needs to fly a distinct distance is characteristic for that ion and lies in the range of 5-20 ms. The time depends on the charge, mass and volume of the molecule as well as the applied electric field and the actual temperature and pressure. To rule out the last mentioned and critical variables, an internal standard is implemented in the measurement, such that the relative time only depends on the molecular characteristics. As a result of the measurement a plasmagram is obtained. Any volatile molecule that can be ionized is detectable by IMS after calibration of the time scale.

The detection limits are about $1 \mathrm{ng}$ for heroin and $0.5 \mathrm{ng}$ for cocaine. Because the nature of the samples is variable and thus the collection of the dust by the vacuum cleaner is not reproducible, quantification is impossible. The results are interpreted as positive or negative with respect to the substance under investigation. The results obtained may be confirmed by extraction of the used filters and analysis of the resulting extract by chromatographic methods.

The IMS technique cannot compete with dogs specially trained to find illicit drugs but it is a useful supplement on the premises.

\section{Sampled Materials}

Clothes are the most frequently sampled material. Typically the dust is only collected from the zipper, the buttons and the pockets - regions that are contaminated solely by the hands of the person wearing these clothes. Dirt under the finger nails is an other very informative object of investigation. If a positive result for an illicit drug is generated, the person must have had direct contact with that substance. Other personal things like glasses, keys, money bags, passports and jewellery are also frequently analyzed. A positive result is most probably due to contamination by the owners' hands. Cars and flats can also be analyzed thanks to the portability of the equipment. Due to the electrostatic attraction of the dust, screens belong to the most promising materials.

Numerous analyses of Swiss banknotes revealed that one third give a positive result for cocaine and about $3 \%$ for heroin. By introduction of cut-off values and standardization of the dust collecting procedure, 'normal currency' becomes distinguishable from 'drug money'. By choosing $1 \mathrm{ng}$ of cocaine and $6 \mathrm{ng}$ of heroin as cut-off values, a single positive result is indicative for cocaine by $90 \%$ and for heroin by $99 \%$ respectively. This probability increases by each positive result in the same investigation [7].

\section{Accreditation}

Some months ago the Swiss Accreditation Service edited the Guidelines for Accreditation of the Swiss Laboratories performing Forensic Drug Analyses in accordance with the Legal Medicine Sector Committee [8]. The corresponding checklist is under preparation. These documents are thought as a tool for the harmonization of the applied procedures in the laboratories performing such drug of abuse testing and should lead laboratories to accreditation. In the near future some of the laboratories intend indeed to make the step to the accreditation of their forensic drug of abuse testing procedures.

\section{Acknowledgements}

The authors wish to thank Mr. Thomas Schwerzmann and Mr. Marco Luginbühl for their technical assistance.

Received: January 22, 2002

[1] Bundesamt für Polizei, Schweizerische Betäubungsmittelstatistik 2000, 'Sicherstellung Heroin und Cocain'.

[2] Gruppe Forensische Chemie der SGRM, 'Richtlinie für die Probenahme von Betäubungsmittel-Asservaten', März 2001.

[3] H. Huizer, Pharmaceutisch Weekblad Scientific Edition 1987, 9, 203-211.

[4] G. Andres, Kriminalistik 2001, 4, 260-263.

[5] SWGDRUGS Recommended Minimum Standards For Forensic Drug Identification, MICROGRAM, Vol. XXXIV, No.6, 150151.

[6] L. Strömberg, L. Lundberg, H. Neumann, B. Bobon, H. Huizer, N.W. van der Stelt, For. Sci. Int. 2000, 114, 67-88.

[7] T. Briellmann, F. Dussy, Kriminalistik 2001, 2, 113-116.

[8] Schweizerische Akkreditierungsstelle, Sektorkomitee Rechtsmedizin, 'Leitfaden zur Akkreditierung von Schweizer Prüflaboratorien zur Durchführung Forensischer Betäubungsmittelanalytik', 2001, Dok Nr. 318 d. 